\title{
Capacity building in optics and photonics by international collaboration
}

\section{Minella Alarcon}

Minella C. Alarcon, "Capacity building in optics and photonics by international collaboration," Proc. SPIE 9663, Eighth International Topical Meeting on Education and Training in Optics and Photonics, 96631M (6 October 2003); doi: $10.1117 / 12.2207504$

SPIE Event: Eighth International Topical Meeting on Education and Training in Optics and Photonics, 2003, Tucson, Arizona, United States 


\title{
Capacity building in Optics and Photonics by international collaboration
}

\author{
Minella C. Alarcon \\ Division of Basic and Engineering Sciences, UNESCO, 1 rue Miollis, 75015 Paris, France \\ Tel.+33 145.68.38.91; Fax+33 1 45.68.58.18;Email: m.alarcon@unesco.org
}

\begin{abstract}
A project seeking to improve capacities in teaching Optics and Photonics in university introductory physics courses and benefit teachers in developing countries will be implemented by international collaboration. Active learning modules in Optics will be based on available resources and inexpensive materials. Most workshop facilitators will come from developing countries.

(ㅇ)2003 Optical Society of America

OCIS codes: (000.2060) Education; (120.4820) Optical Systems
\end{abstract}

\section{Introduction}

UNESCO is the only international organization with a mandate in capacity building in science and technology. Within this context, UNESCO has existing programmes in physics, mathematics, chemistry and the life sciences. Projects and initiatives in university physics education are implemented under the physics programme. It is within this framework that UNESCO recently launched a project aiming primarily to improve capacities in teaching optics and photonics in introductory physics courses in the university.

The purpose of this paper is to discuss the work of UNESCO in promoting physics education in developing countries and how this experience is applied to optics and photonics.

UNESCO usually implements its projects in physics education through international collaboration, with the participation of as many countries as practically possible. UNESCO fosters capacity building for physics research through its continuing support of the Abdus Salam International Centre for Theoretical Physics (ICTP), well known for its activities organized for the benefit of physicists from developing countries. As well, UNESCO was instrumental to the establishment of the International Commission of Physics Education (ICPE), otherwise known as Commission 14 of the International Union of Pure and Applied Physics. For the specific purpose of promoting physics education, regional physics education networks were established under the auspices of UNESCO in the 1980's- the Asian Physics Education Network (ASPEN), the Arab-African Physics Education Network (ARAPEN), and the Latin American Physics Education Network (LARAPEN). For more than 10 years, these networks saw the involvement of many countries in various UNESCO activities in physics education. Of these networks, only ASPEN survived the changing and difficult times in the different regions of the world.

In 1990, UNESCO launched a project called University Foundation Course in Physics (UUFCP) [1], which aimed to strengthen through international cooperation, university teaching at the introductory level in physics. It proposed to develop a new approach for the introductory physics course by bringing together expertise from developing and developed countries. It was designed to assist universities, especially those in developing countries, in their efforts to improve the quality, effectiveness, and relevance of the introductory physics courses. While emphasizing the need for more laboratory work and topics on contemporary physics, UUFCP recognized that not all topics can be taken up with equal rigor and effectiveness. Through ASPEN, with participating experts from China, India, Japan, Australia and the Philippines, the project took off in Asia and resulted in the production of a textbook, modules on selected topics in contemporary physics, laboratory manuals, instructional materials on video and computer simulation software.

UUFCP is significant because it was a pioneering effort to break away from the traditional approach in the way introductory physics was taught. Moreover, it enabled different countries, through international collaboration, to develop their own instructional materials.

With support from UNESCO, ASPEN activities have been organized to respond to the following problems in university physics education [2]:

1) The lack of creativity and innovation in the way physics is taught, resulting in boring chalk-\&-talk classroom lectures and the students losing interest in physics;

2) The need for upgrading and providing foundation to the conceptual knowledge of many physics teachers and lecturers, especially those in developing countries. 
ASPEN has responded to these problems mainly through conferences and workshops on topics including undergraduate laboratory, use of demonstrations, importance of conceptual understanding, and the use of computers in physics experiments.

\section{Active learning in Physics}

Since 1996, UNESCO and ASPEN organized more activities featuring hands-on workshops and innovations resulting from physics education research mainly from physics departments in the United States. In particular, the active learning technique has been the topic of recent workshops, as well as that of curriculum material development, building regional capacities in the use and application of the method. Workshops have been run in Laos, Malaysia, the Philippines, South Korea, Sri Lanka, Vietnam, and very recently, in Ghana in Africa. Fig. 1 shows a typical workshop in Sri Lanka. Moreover, with UNESCO support, active learning modules on topics in mechanics have been developed and compiled for free distribution [3]. An illustration from one of these modules is reproduced in Fig. 2.

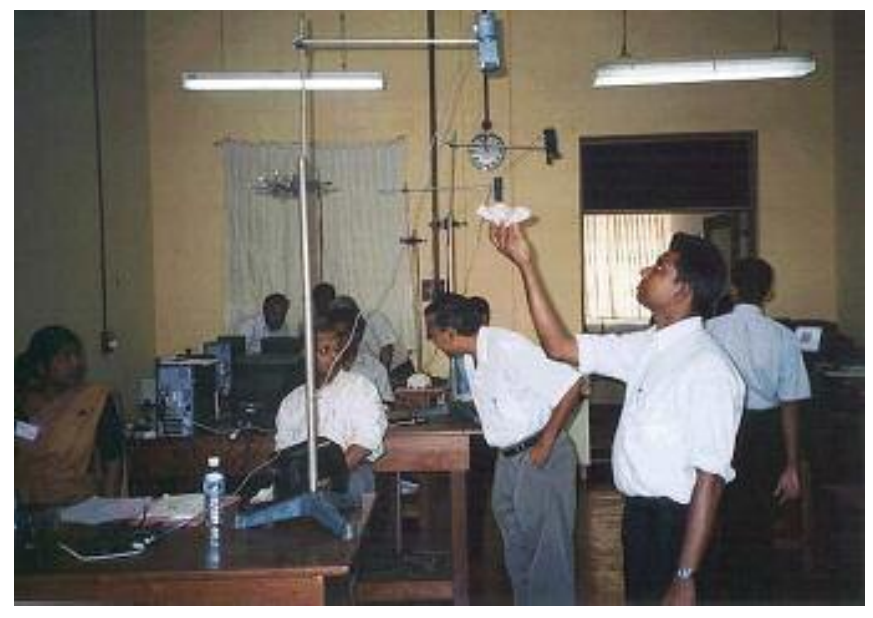

Fig. 1. An active learning workshop in Sri Lanka.

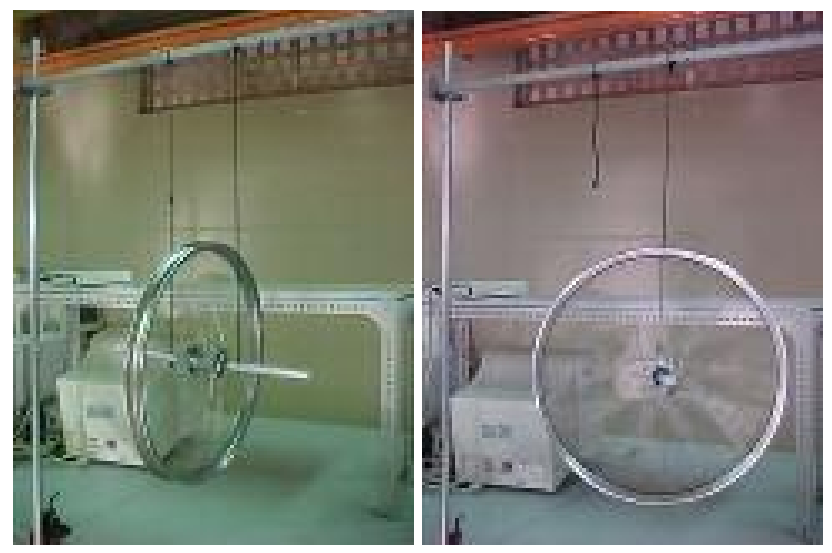

Fig. 2. An active learning module on angular momentum: what happens to the rotating wheel if one of the strings is cut?

The active learning method, also known as 'interactive engagement,' cares about the student and her/his learning style and endeavors to match the teacher's strategy with the student's learning style. The active learning environment is characterized as student-centered and activity-based using computers and equipment. Lectures are kept to a minimum. Students are actively engaged or participating actively in the learning process. When students, working in groups, are actively engaged with class experiments, for example, and guided to make observations, experiment, make mathematical descriptions, and construct theories, they develop scientific reasoning skills and learn the underlying principles and concepts [4]. On the other hand, the instructor prepares the learning environment, 
and through activities, questions, discussions, guides students through the reasoning necessary to construct concepts, then apply them to real-world situations [4]. Finally, assessment and evaluation are considered an essential part of the active learning method [5]. It is important to determine using suitable instruments how well the students are learning physics concepts.

Active learning in physics is being developed through physics education research, applying the rules of evidence of experimental physics, with the purpose of promoting active mental engagement of students in the process of learning physics. Much of the research has focused on the transformation of passive instruction in large lecture halls with large numbers of students, the usual setting of many physics classes. Interactive engagement has been shown to have successfully resulted from, to mention a few, the use of short lectures interspersed with conceptual multiple-choice questions fostering student-instructor interaction [6], the use of interactive lecture demonstrations supported by real-time microcomputer-based laboratory tools [7], the use of materials with carefully structured experiments, exercises and questions that guide students step-by-step through reasoning in constructing concepts [8], and the use of a series of related activities designed to follow a learning sequence and using apparatus and computer tools [9]. The research-based development of curriculum has led to the development of standardized assessment instruments designed to probe conceptual understanding. Some of these tools are notably the Force Concept Inventory (FCI), the Force and Motion Conceptual Evaluation (FMCE), and the Conceptual Survey of Electricity and Magnetism (CSEM) [5].

In an active learning environment, the instructor gives up her/his role as the source of all information in the classroom to give way to being guide and facilitator [10]. This is perhaps the biggest challenge there is in adopting this method of teaching since most teachers must have been taught in traditional passive learning environments. On the other hand, the students cease to be mere receivers of information and are no longer subject to mindless notetaking in the classroom [10]. A carefully designed instrument for assessment of student learning is an integral part of the learning process [8]. This assessment tool is different from the usual examination problems requiring manipulation of formulas or questions that can be answered on the basis of memorization.

UNESCO and ASPEN promote the active learning method, especially in developing countries, for a number of reasons. Together, UNESCO and ASPEN have consistently promoted the use of laboratory and hands-on activities in physics classes. Research results show that implementing an activity-based curriculum and mentally engaging students in class can promote conceptual understanding. The method encourages the physics instructor to do research and enable her/him to devise and find creative ways to improve her/his teaching and student learning. This is one research area where physics teachers from developing countries can make valuable contributions. Physics education research may provide the key to student learning in physics and needs to be widely fostered by the international physics community.

\section{Training trainers in Optics and Photonics}

By promoting the adoption of the active learning method, UNESCO aims to improve capacities in teaching optics and photonics in introductory physics courses in the university.

The project is focused on optics and photonics because it is one of the few experimental physics areas that have been found to be relevant and adaptable to research and educational conditions in many developing countries. It is one research area that is closely linked to industrial applications, and has been found useful in a number of developing countries, notably in Southeast Asia and Africa. Optics and laser research laboratories can be found in Indonesia and the Philippines, to name a few, as well as in Ghana, Tunisia and Senegal. Experiments in optics and photonics are relatively inexpensive and cost-effective to set up and can be implemented in a modular manner. Most often, space and power requirements are reasonable and affordable. Moreover, with the advances in optical communications and other laser applications in industry and medicine, some level of local research collaboration with industries and hospitals has become possible. Furthermore, opportunities for regional research collaboration exist for photonics applications to environmental problems of regional scope. In general, the challenge for research for experimental physicists in developing countries is finding an application relevant to the local needs and existing conditions, and the corresponding logistical support.

The project is proposed for the benefit of physics teachers from developing countries. It hopes to encourage them to teach better the optics part of the introductory physics course by drawing examples from local research activities and inspire students to do projects and pursue further studies in the field. Fostering understanding and appreciation of optics in high school and the early years after would also lead to better training of technicians. Consequently, this may lead to an increase in public understanding and interest in the important benefits derivable from applications of optics and photonics. In the long run, public realization of the significance of local optics and photonics research may open up opportunities for funding and logistical support. 


\subsection{Objectives of the project}

The project seeks to promote the teaching of optics in the level of introductory physics in the university. The project encourages physics teachers to take up topics in geometrical and wave optics and use the active learning method in the process. Teachers will be trained to become active learners themselves. Active learning workshops will enable teachers to design and prepare various class activities, such as simple experiments, demonstrations, exercises, using available materials and equipment. Teachers will practice making predictions and formulating questions that will probe and guide students through the reasoning necessary to explain observations and experimental results. The project seeks to promote physics education research by using the teacher-designed activities to investigate student understanding of basic optics principles and concepts. Teachers will be encouraged to do literature research and be familiar with background information and published research results, as well as document their own procedures and results.

Teachers will be trained to formulate and use instruments to assess student understanding. Most of these tools are qualitative questions that are administered before (pretest) and after (posttest) a topic or a course is taken up with the students. In his comparison of the effectiveness of traditional versus interactive engagement methods in mechanics, Hake [5] used the normalized gain (gain/maximum possible gain). While the normalized gain may provide a good measure of the effectiveness of the teaching strategy used, Hake cautions that the normalized gain does not give a definitive assessment of the overall effectiveness of the method. While some research results from work done on probing conceptual understanding of optical principles have been published, a standardized assessment instrument on optics still has to be put together.

Finally, the project seeks to develop through physics education research creative and innovative teaching and learning materials in optics and photonics, as well as instruments for assessment. Teachers will be encouraged to document their own procedures and results, conduct systematic investigation of student understanding of optics by designing activities and formulating probing questions with clear objectives and expected outputs, formulate appropriate tools and assess the effectiveness of the method, and use the results to improve further the application of the method. The process may lead to the identification of learning difficulties and misconceptions, which can be analyzed and addressed through research.

Training teachers to become active learners and apply the method in their teaching should provide foundation to their own conceptual knowledge. Hestenes [11] defines "conceptual learning" as opposed to "rote learning," which depends on memorization of facts and formulas, and explains it in several ways.

Conceptual learning is a creative act. The teacher who understands well the basic optics concepts will be able to design varied class activities and formulate engaging guide questions. The student who has a good grasp of basic optics concepts will enjoy and look forward to doing class activities and pursuing projects. An active learner will therefore foster creativity and innovations.

Conceptual learning is systemic. Concepts derive their meaning from a coherent conceptual framework. Interference and diffraction of light are defined within the context of the wave model of light. Active learners should be able to see and understand the interrelationships among physical quantities within the appropriate framework.

Conceptual learning is contextual. The active learning method will be introduced in developing countries in ways recognizing this fact.

\subsection{Active learning in Optics}

A reference database is being compiled of what is available in the literature on the topic. This section presents two examples from literature of physics education research results in optics.

The first example is taken from the project Constructing Physics Understanding in a Computer-Supported Learning Environment (CPU) and focuses on image formation by converging lenses [12]. Research has shown that students have difficulty understanding the following ideas:

(1) From each point source, light travels outward in all directions.

(2) An extended light source can be considered as a sequence of source points.

(3) In the image formation process light spreading out from each point on the source passes through the converging lens and comes together at a unique point called the image point.

To help students better understand these ideas, CPU curriculum materials and simulators were designed. Students can use the CPU light and color simulator to construct set-ups with a light source, lens and screen that match the laboratory experiments. The simulator can reproduce whatever appears on the screen and generate light ray diagrams. 
In the actual experiment, a MiniMaglite flashlight is used to approximate a point source in front of a lens and a screen. On the simulator, a point source is used with a light ray spray tool to generate light rays emerging from the point source through the lens that focuses the light rays on a unique point on the screen. In the laboratory set-up, when the screen is moved closer or further from the lens, students can observe on the simulator that blurring images correspond to the screen intercepting light rays before or after converging to a single point. Several MiniMaglites in front of the lens and screen are used to enable students to imagine a sequence of point sources. The simulator displays the analogous situation. Finally, students do both laboratory and simulator experiments with an extended light source. As the student traces points on the extended light source from one end to another, he can see the corresponding image points mapped out on the simulator. The student then directly observes that each image point corresponds to a point on the source.

The Physics Education Group at the University of Washington has been engaged in a long-term investigation of student understanding of geometrical and physical optics. The second example is taken from a paper reporting their investigation of student understanding of double-slit interference of light [13]. The context for the group's research is during a 50-minute tutorial attended by 20-24 students working collaboratively in groups of 3 or 4 . Designed to supplement lectures and the textbook, tutorials emphasize constructing concepts, developing reasoning skills, and relating physics formalism to the real world. Each tutorial session consists of a pretest, worksheet, homework assignment, and a posttest.

In investigating student understanding of double-slit interference of light, interviews and responses to questions indicate that it is necessary to develop a basic wave model for the phenomenon. To do so, exercises with the ripple tank were used to demonstrate interference properties of water waves. Waves in the ripple tank are easier to observe than light waves and make it possible for the students to visualize wave fronts. Through interviews and pretests, student difficulties in understanding the aspects of the problem are exposed. With respect to developing a wave model for double-slit interference of water waves, students had difficulty relating interference to path difference, and understanding how principle of superposition applied to water waves. The tutorial is then designed to guide the students to recognize the problem and help them go through the reasoning required to resolve the difficulty. In this case, the students are given a diagram of two overlapping sets of concentric circles representing wave fronts due to two point sources of water waves, and tutorial questions help them analyze the phenomenon step-by-step. A homework assignment reinforces their observations in the tutorial exercises and helps the students derive and apply formulas for locating nodal lines and lines of maximum constructive interference.

The next step is to establish the analogy between superposition in water and in light waves. This is done by addressing the failure of the students to relate a sufficiently narrow slit to a point source. A tutorial exercise is designed to make students arrange sponges in a shallow pan of water to form slits of various widths. They generate parallel wavefronts and observe what happens when the waves pass through sufficiently wide and narrow slits. The tutorial then guides the students in making a proper analogy between interference in water and in light. Moreover, the tutorial on double-slit interference includes an exercise designed to help students recognize that geometrical optics cannot be used to make predictions about light passing through very narrow slits. Finally, using a pretest to expose the difficulty, tutorial exercises are used to help students recognize how a two-slit interference pattern is affected by changes in the slit separation and other parameters of the optical system.

The paper goes on to describe the work on extending the model to multiple slit interference, single slit diffraction, and so on. The authors emphasize that the improvement gained by the students in understanding the wave model for double slit interference of light is reinforced by extending the model to other cases and building on their experience from the double slit case.

\subsection{Implementation of the project}

The project will be implemented by an international working group whose members have expertise in optics and photonics and in the active learning method. As a project proposed for the benefit of physics teachers in developing countries, it is important that they are well-represented in the working group. Building on its experience of continuing cooperation with ASPEN, UNESCO has began to promote active learning in other regions, particularly Africa, in order to address similar issues in physics education. ASPEN is expected therefore to play an active role in the project, and so will physics teachers from Africa. ASPEN representatives in the working group will come from countries that have given efforts to adopt the active learning method in their physics classes. They are ASPEN members who have designed demonstrations and simple experiments and are applying them in their physics classes. As the UNESCO institute promoting physics research and providing consistent support to optics and photonics researchers in developing countries, ICTP is involved in the project implementation. The international optics community is represented in the project working group by one OSA and one SPIE officers. UNESCO is providing 
seed funds for the initial implementation. While the project working group is kept deliberately small in size, participation of more people in the implementation will be invited in due time.

The project has started by providing modest support to ASPEN members from the Philippines to develop active learning modules in selected topics in optics. These curriculum materials will make use of simple optical elements and other inexpensive components, as depicted in Fig. 3. The modules will be used in the project to introduce and demonstrate the active learning method to the project working group, and to initiate research in the Philippines of student understanding of basic optical principles.

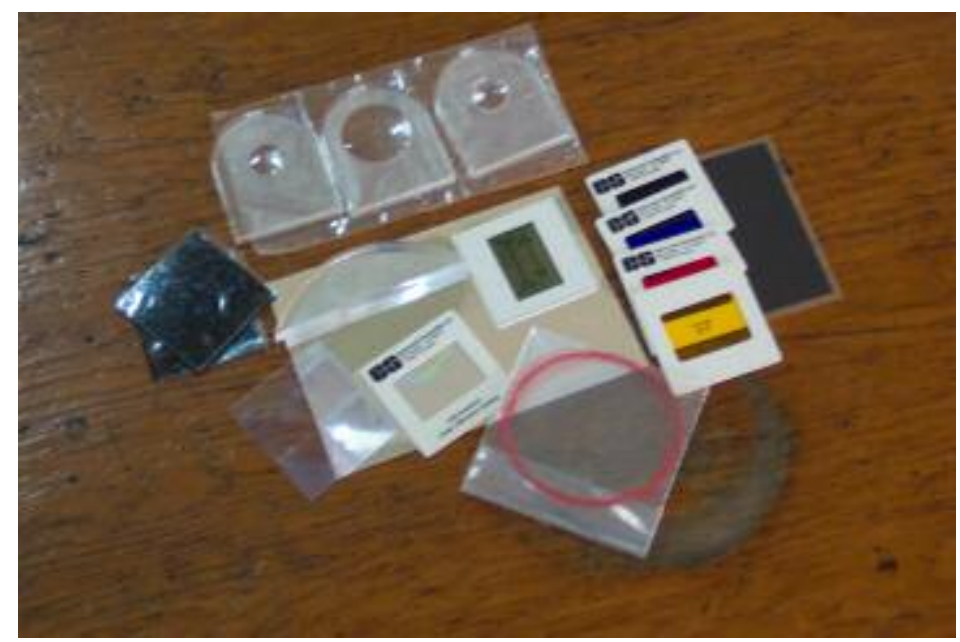

Fig. 3. Some simple optical components to be used in active learning curriculum development.

The project focuses on the active learning method, the student-centered, hands-on, minds-on approach to introductory physics teaching. Teaching optics the active learning way implies the use of a variety of class activities where the students are actively engaged and the instructor acts as guide and facilitator. How different will these teaching and learning materials be from those that have been described in the literature? McDermott [8] emphasizes that a lot of time and effort goes into development of curriculum that is effective and can be used by different instructors. Her advice is "to take advantage of existing curriculum that has been thoroughly evaluated." By considering the diverse needs and conditions existing in target countries, learning and teaching curriculum in optics are to be developed based on available resources and the cultural context. Materials already developed and evaluated elsewhere within a specific context will certainly be valuable. However, they have to be adapted to existing conditions if they are to be effectively applied in a different setting. The outstanding work of the pioneers of physics education research serves as a model to be followed by other physics teachers. Their commitment and hard work are a source of inspiration and serve to awaken the inherent creativity of many physics teachers, especially those from developing countries.

While assuming that the project beneficiaries may have been taught in passive learning environments, there are many factors to be considered in context-based active learning curriculum development. A few are enumerated as follows:

1) the way the introductory physics course is taught at present in universities; textbooks and references available; language used in instruction; is the local language used in schools and universities?

2) state of physics teaching laboratories; available materials and equipment, including computers, as well as repair and maintenance facilities; feasibility of local acquisition of new equipment, by purchase or fabrication

3) condition of buildings and rooms; space available for physics teaching

4) number of students, men and women, in physics classes at various levels; their science background prior to university; language/s spoken

5) local efforts to improve physics teaching

6) level of government or public support for science education and research

7) salaries and compensation for teachers

Most workshop facilitators and resource persons for the project will come from developing countries and more of them will be trained, as was done in Fig. 4. The trainees in the workshop shown in the figure were presented with all kinds of available devices and materials, both computer and non-computer-based. 


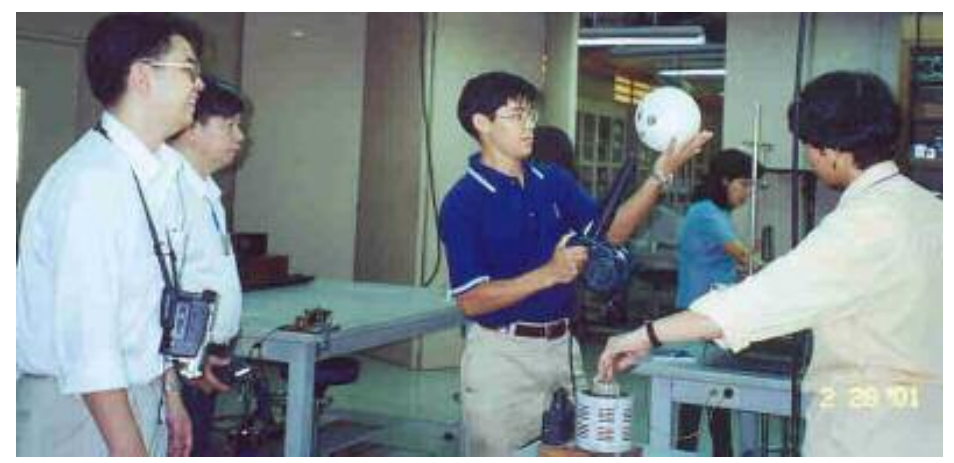

Fig.4. UNESCO-ASPEN trainers' workshop in active learning in physics [10]

Optics trainers' training courses are expected to be similar to previous UNESCO-ASPEN active learning workshops where modern optical devices, when they are available, can be used together with traditional equipment and inexpensive materials. Training organizers will be encouraged to cooperate with local optics and photonics researchers and take advantage of their expertise and experience. Optics teachers will be encouraged to draw examples from local research activities. Various kits of simple optical components will be tried out and evaluated. Training participants will be given time to familiarize themselves with, play and experiment with the equipment, to enable them to design and develop teaching and learning materials.

The following activities are foreseen as important components of the project implementation:

1) Preparation of active learning trainers' training syllabi, materials and equipment in optics and photonics

2) Trainers' training workshops in different countries

3) Developing teaching and learning materials (kits) adapted to different conditions in participating countries.

4) Developing instruments for assessment and evaluation in optics and photonics.

\section{Challenges to the project [2]}

A challenging project is always worthwhile to pursue. The most important challenge of all is learning to become an active learner. The shift from a passive learning environment to an active learning one is not easy for both student and teacher. For the teacher, he/she needs to give up lectures and give way to being a guide and facilitator. For the students, they need to appreciate the involvement required from them.

Applying the active learning strategy requires advance planning and preparation of the instructional and learning materials, on the part of the instructor, and reviewing material and doing homework regularly, on the part of the students. For some students and teachers, this may seem an unreasonable demand for time and energy.

An activity-based curriculum takes much time to implement in the classroom. This implies that less topics can be covered in depth within a given term or semester. This may seem frustrating to most teachers who feel that a complete introductory optics course should cover all topics in geometric and physical optics with equal rigor and depth. The issues of coverage in optics should be discussed as a community including both teachers and researchers.

Equally daunting is the task of adapting the technique to the characteristics, the needs and the problems of the situation and the people involved. The active learning method should be applied in consideration of the varied contexts of its users. Adaptation calls for creativity in implementing the strategy, sensitivity towards needs and difficulties, and flexibility in handling unfamiliar situations. Active learning was first introduced to ASPEN with the exclusive use of computers and computer-interfaced sensors and measuring devices. Being characterized by great diversity, Asia has universities and physics departments endowed with facilities ranging from the most advanced to the oldest and most traditional. Colleagues from less endowed universities only had traditional equipment. For them, the ASPEN active learning team developed non-computer-based interactive lecture demonstrations, and proved that traditional physics equipment and inexpensive locally-available materials can be used to promote conceptual understanding.

It must be recognized that an activity-based curriculum has greater demands for resources than the traditional lecture-based course. It is not easy to find these resources.

It is important to have a group of resource persons coming from a certain region that identifies with the workshop beneficiaries and understands the conditions in the region. Having a regionally indigenous resource group 
would encourage ownership of the strategy and quickly build confidence among colleagues. Training resource persons may prove to be a great challenge, since the number of physics teachers inadequately trained in the discipline is quite daunting. Trainers' training workshops then should be regarded as an opportunity to upgrade the conceptual knowledge of many university and secondary school physics teachers and lecturers.

The cultural diversity in a given region lends itself to an important issue that is reflected in varied learning styles of students. For example, while students in the Philippines can be easily encouraged to come forward and discuss the results of their measurements, this may not be necessarily so for students in South Korea. This is a definite challenge to the instructor, who is expected to be creative in exploring ways of actively engaging students through class activities and discussions.

Finally, there is a need to keep up with new developments in physics education research and share experiences, information and materials. Primarily, this implies the need to access journals and research literature, and contribute articles. This is part of the bigger issue of dissemination of scientific knowledge to developing countries. On one hand, there is a need to provide access to the increasing number of research papers addressing different issues in physics education. On the other hand, as physics teachers succeed in adapting innovations, produce materials and gain experiences, they will find the need to document their work and results, and share them with fellow researchers in the field. The physics teachers from developing countries, the beneficiaries of this project, should be prepared for this eventual development.

Some of the challenges mentioned are a consequence of the pervading traditional culture in physics teaching and are expected to be resolved, as the project progresses and the overall situation of physics education improves.

Many of these challenges may be overcome through collaboration and networking. Physics teachers can work together, exchange ideas and experiences, learn from one another, and together endeavor to find the many resources required in achieving the paradigm shift in physics education.

\section{References}

M.C. Alarcon, "Recent innovations in university introductory physics teaching," AAPPS Bulletin, 9, 23-26 (1999).

M.C. Alarcon, "The challenges of adapting active learning in physics to the cultural context," in Proceedings of the UNESCO-ASPEN Workshop on Active Learning in Physics, Sri Lanka, 2-4 December 2002.

O.L. Cambaliza, I.B. Culaba, and J.T. Maquiling, ASPEN-UNESCO Active Learning Classroom and Laboratory Activities, in CD-ROM (2002).

P. W. Laws, "Calculus-based physics without lectures," Phys. Today 44, 24-31 (1991).

R.R. Hake, "Assessment of physics teaching methods," in Proceedings of the UNESCO-ASPEN Workshop on Active Learning in Physics, Sri Lanka, 2-4 December 2002.

D.E. Meltzer, "Transforming the lecture-hall environment: the fully interactive physics lecture," Am. J. Phys. 70, 639-654 (2002).

R.K. Thornton and D.R. Sokoloff, "Assessing student learning of Newton's laws: the force and motion conceptual evaluation and evaluation of active learning laboratory and lecture curricula," Am. J. Phys. 66, 338-352 (1998).

P. W. Laws, "Millikan Lecture 1996: Promoting active learning based on physics education research in introductory physics courses," Am. J. Phys. 65, 14-21 (1997).

L. C. McDermott, "Oersted Medal Lecture 2001: Physics education research - the key to student learning," Am. J. Phys. 69, 1127-1137 (2001).

O. L. Cambaliza, A. P. Mazzolini, and M. C. Alarcon, "Adapting active learning approaches in physics education to local Asian environments," in ICPE International Conference on Teaching and Learning of Physics in Cultural Contexts, Korea, 13-17 August 2001.

D. Hestenes, "Oersted Medal Lecture 2002: Reforming the mathematical language of physics," Am. J. Phys. 71, 104-121 (2003).

F. Goldberg, "The CPU Light and Colour Computer Simulators," Synopsis of paper presented at 2001 meeting of the European Science Education Research Association, Thesolloniki, Greece, http://www.sci.sdsu.edu/CRMSE/fgoldberg.html.

K. Wosilait, P.R.L. Heron, P.S. Shaffer, and L.C. McDermott, "Addressing student difficulties in applying a wave model to the interference and diffraction of light,” Phys. Educ. Res., Am. J. Phys. Suppl. 67, S5-S15 (1999). 